\title{
Modulation of Myostatin/Hepatocyte Growth Factor Balance by Different Hemodialysis Modalities
}

\author{
Pasquale Esposito, ${ }^{1}$ Edoardo La Porta, ${ }^{1}$ Marta Calatroni, ${ }^{1}$ Maria Antonietta Grignano, ${ }^{1}$ \\ Samantha Milanesi, ${ }^{1}$ Daniela Verzola, ${ }^{2}$ Yuri Battaglia, ${ }^{3}$ Marilena Gregorini, ${ }^{1}$ \\ Carmelo Libetta, ${ }^{1}$ Giacomo Garibotto, ${ }^{2}$ and Teresa Rampino ${ }^{1}$
}

\author{
${ }^{1}$ Department of Nephrology, Dialysis and Transplantation, Fondazione IRCCS Policlinico San Matteo and University of Pavia, \\ Pavia, Italy \\ ${ }^{2}$ Department of Internal Medicine, Istituto Nazionale per la Ricerca sul Cancro, University of Genoa and \\ IRCCS Azienda Ospedaliera Universitaria San Martino-IST, Genoa, Italy \\ ${ }^{3}$ Nephrology and Dialysis Unit, St. Anna University Hospital, Ferrara, Italy
}

Correspondence should be addressed to Pasquale Esposito; pasqualeesposito@hotmail.com

Received 19 January 2017; Accepted 20 March 2017; Published 28 March 2017

Academic Editor: Milton O. Moraes

Copyright $\odot 2017$ Pasquale Esposito et al. This is an open access article distributed under the Creative Commons Attribution License, which permits unrestricted use, distribution, and reproduction in any medium, provided the original work is properly cited.

\begin{abstract}
Background. In this study we investigated the relevance of myostatin and Hepatocyte Growth Factor (HGF) in patients undergoing hemodialysis HD and the influence of different HD modalities on their levels. Methods. We performed a prospective crossover study in which HD patients were randomized to undergo 3-month treatment periods with bicarbonate hemodialysis (BHD) followed by online hemodiafiltration (HDF). Clinical data, laboratory parameters, and myostatin and HGF serum levels were collected and compared. Results. Ten patients and six controls (C) were evaluated. In any experimental condition myostatin and HGF levels were higher in HD than in C. At enrollment and after BHD there were not significant correlations, whereas at the end of the HDF treatment period myostatin and HGF were inversely correlated $(r-0.65, p<0.05)$, myostatin serum levels inversely correlated with transferrin $(r-0.73, p<0.05)$, and HGF levels that resulted positively correlated with BMI $(r 0.67, p<0.05)$. Moving from BHD to HDF, clinical and laboratory parameters were unchanged, as well as serum HGF, whereas myostatin levels significantly decreased $(6.3 \pm 4.1$ versus $4.3 \pm 3.1 \mathrm{ng} / \mathrm{ml}, p<0.05)$. Conclusions. Modulation of myostatin levels and myostatin/HGF balance by the use of different HD modalities might represent a novel approach to the prevention and treatment of HD-related muscle wasting syndrome.
\end{abstract}

\section{Introduction}

Patients suffering from chronic kidney disease (CKD), mainly those undergoing hemodialysis (HD), often present malnutrition and muscle wasting syndrome, which directly correlate with morbidity and mortality [1]. Several mechanisms have been involved in the regulation of energy and muscular homeostasis, including cytokines and molecules with systemic and paracrine action (the so-called "myokines") and intracellular signalling pathways, that may have positive or negative effects on muscle growth, also affecting muscle response to injury through the inhibition or stimulation of muscle stem cells ("satellite cells") [2]. The Growth Differentiation Factor-8 (GDF-8)/myostatin, a member of
TGF- $\beta$ family, is primarily expressed in skeletal muscle and has the effect of limiting muscle growth. It also circulates in the blood and acts on muscle tissue, binding a cellbound receptor called Activin type 2B [3]. The importance of myostatin was emphasized by several studies, demonstrating its increase in muscular atrophy and chronic disease [4-6].

The Hepatocyte Growth Factor (HGF) is another key factor in the regulation of skeletal muscle homeostasis. HGF was firstly recognized as a product of mesenchymal cells with an action predominantly addressed to stimulate growth, motility, and differentiation of epithelial-derived cells [7]. Subsequently, it has been demonstrated that HGF is also present in muscle tissue and is essential for the response to cellular damage and the repair of damaged tissue, inducing activation and 
rapid cell division of muscle satellite cells [8]. In CKD patients an upregulation of myostatin gene expression in skeletal muscle has been found, which was strictly related to IL-6 expression, suggesting a link between myostatin and microinflammation [9]. Moreover, it has been demonstrated in the clinical setting that myostatin serum levels in HD patients are elevated and inversely related to muscle strength [10].

Similarly, also HGF levels appear to be influenced by uremia and HD. Indeed, it has been proved that HD causes a prompt and prolonged release of HGF into the circulation, mainly because of the leukocyte activation associated with HD treatment [11].

Therefore, considering their elevated circulating levels and apparently opposite effects on muscular metabolism, it is conceivable that myostatin and HGF accumulation, as well as their balance, may be important in the pathogenesis of malnutrition and muscle wasting syndrome in uremic subjects.

This is the reason why we decided to investigate the relevance of myostatin and HGF in HD patients and whether their serum levels could be modulated by using different HD modalities.

\section{Patients and Methods}

We performed a prospective 6-month crossover study, enrolling clinically stable uremic patients undergoing standard bicarbonate hemodialysis (BHD) at least for 6 months. Patients with acute infections, active immunological diseases, immunosuppressive therapy, previous transplantation, or history of malignancies were excluded from the study. Sexmatched healthy subjects were the control group. Patients were randomized into two groups according to a $2 \times 2$ crossover design. Group 1 was treated with BHD for the first 3 months and then switched to online hemodiafiltration (HDF) for additional 3 months; group 2 was initially treated with HDF and then with BHD.

BHD was performed with cellulose diacetate membranes (DICEA, Baxter Healthcare, Baxter, Iowa, USA) with a blood flow of $300-350 \mathrm{ml} / \mathrm{min}$ and a dialysate flow of $600 \mathrm{ml} / \mathrm{min}$. In $\mathrm{HDF}$, the replacement volume was standardized to $25-30 \%$ of the total treated blood volume, using a high-flux Helixone membrane (FX100, Fresenius Medical Care, Bad Homburg, Germany).

At the beginning of the study (enrollment phase) and at the end of each experimental period we evaluated anthropometric and nutritional parameters, including body mass index (BMI), predialysis serum levels of phosphate, albumin, transferrin, lymphocyte count, and blood urea nitrogen, and dialysis adequacy (evaluated as single pool KT/V-spKT/V). In each patient, before the hemodialysis session, serum was withdrawn and tested for HGF and myostatin levels by ELISA (Quantikine; R\&D Systems, Minneapolis, MN, USA; detection limit $40 \mathrm{pg} / \mathrm{ml}$ and $5.3 \mathrm{pg} / \mathrm{ml}$, resp.), assuming those samples presenting values under the detection limit as zero.

The study was conducted in accordance with the Declaration of Helsinki and was approved by the Ethics Committee of the Fondazione IRCCS Policlinico San Matteo of Pavia, Italy. Written informed consent was obtained from each participant prior to enrollment in the study.
2.1. Statistical Analysis. Quantitative variables were represented by mean \pm standard deviation (SD) or interquartile ranges (IQR) if they were not normally distributed (Shapiro Test).

Differences among control subjects and HD patients in different experimental conditions were assessed by analysis of variance (ANOVA), Student's $t$-test, or nonparametric Mann-Whitney test when appropriated. In order to evaluate a possible carry-over effect, we compared the variations of myostatin and HGF levels between subjects of group 1 (i.e., starting with BHD) versus group 2 (i.e., starting with $\mathrm{HDF}$ ). Correlations among myostatin, HGF levels, and variables were analyzed with Spearman-Rho. All tests were two-sided and $p<0.05$ was considered statistically significant. Data analysis was performed with GraphPad Prism statistical package (version 5.00, GraphPad Software, San Diego, California, USA).

\section{Results}

3.1. Patient Characteristics. We enrolled ten patients $(65.5 \pm$ 13.1 years, seven males) with a dialysis vintage of $70.8 \pm 18$ months.

Mean BMI was $28.4 \pm 4.7 \mathrm{~kg} / \mathrm{m}^{2}$; three patients were diabetic. At the time of enrollment all patients were undergoing thrice-weekly 4-hour BHD, with spKT/V of $1.46 \pm 0.4$.

Six healthy subjects $(48.1 \pm 12.7$ years, four males; BMI $26.5 \pm 2.3 \mathrm{~kg} / \mathrm{m}^{2}, p=0.3$ versus patients) constituted the control group (C). After randomization, five patients (50\%) underwent BHD as initial treatment (group 1) and five received $\mathrm{HDF}$ (group 2).

3.2. Myokine Profile. At enrollment and during the two different experimental conditions (BHD or HDF) myostatin levels in patients resulted higher than in $C$, without reaching statistical significance (at enrollment $6 \pm 3.4$ versus $3.1 \pm$ $0.6 \mathrm{ng} / \mathrm{ml}$, resp., $p=0.07$ ).

On the opposite, HGF resulted under the detection limit in $\mathrm{C}$ and four HD patients, reaching levels of $150.5 \mathrm{pg} / \mathrm{ml}$ (IQR 44.2-681) in the remaining six HD patients.

The correlation analysis performed on data collected at each experimental phase showed that, at enrollment and the end of BHD period, myostatin levels did not correlate with any clinical (age, dialytic vintage, and BMI) and laboratory (albumin, transferrin, phosphorus, and calcium) parameter. On the contrary, myostatin levels measured at the end of HDF period resulted in being inversely correlated with transferrin $(r=-0.73, p<0.05)$ and HGF levels $(r=-0.65, p<0.05)$.

Interestingly, similar results were obtained for HGF levels. Indeed, while HGF did not correlate with any variable at the enrollment and after $\mathrm{BHD}$, it resulted in being positively correlated with BMI $(r=0.67, p<0.05)$ at the end of HDF treatment.

3.3. Effect of Different Dialytic Treatments. During the two different treatment periods, there were no significant changes in clinical and laboratory parameters (Table 1).

After shifting from BHD to HDF, HGF levels remained unchanged [BHD 93 (IQR 42-231.8) versus HDF 156.7 (IQR 
TABLE 1: Laboratory and dialysis parameters in patients at enrollment and at the end of each treatment period with different dialysis modalities.

\begin{tabular}{|c|c|c|c|c|}
\hline & Enrollment & $\mathrm{BHD}$ & $\mathrm{HDF}$ & $p$ \\
\hline BMI $\left(\mathrm{kg} / \mathrm{m}^{2}\right)$ & $28.4 \pm 4.7$ & $28.8 \pm 4.8$ & $28.9 \pm 5.1$ & 0.5 \\
\hline Serum albumin (g/dl) & $3.8 \pm 0.3$ & $3.7 \pm 0.2$ & $3.8 \pm 0.2$ & 0.5 \\
\hline C-RP (mg/dl) & $0.47(9.3-0.67)$ & $0.6(0.3-1)$ & $0.45(0.3-0.77)$ & 0.3 \\
\hline Transferrin (mg/dl) & $157.8 \pm 15.1$ & $167.7 \pm 42.3$ & $172.2 \pm 32.1$ & 0.2 \\
\hline Phosphate (mg/dl) & $4.4(3.8-5.7)$ & $4.7(4.3-5.3)$ & $4.1(3.9-4.7)$ & 0.5 \\
\hline Calcium $(\mathrm{mg} / \mathrm{dl})$ & $9 \pm 0.3$ & $9.3 \pm 0.6$ & $9.2 \pm 0.5$ & 0.6 \\
\hline PTH (pg/ml) & $210(149-359)$ & $173.9(106-599)$ & $227(156-361)$ & 0.3 \\
\hline Lymphocyte count & $1283 \pm 673$ & $1255 \pm 588$ & $1396 \pm 567$ & 0.5 \\
\hline BUN (mg/dl) & $71.4 \pm 8.6$ & $80.2 \pm 18.5$ & $77.2 \pm 13.1$ & 0.4 \\
\hline $\mathrm{spKT} / \mathrm{V}$ & $1.46 \pm 0.4$ & $1.36 \pm 0.25$ & $1.42 \pm 0.2$ & 0.4 \\
\hline Predialysis myostatin $(\mathrm{ng} / \mathrm{ml})$ & $6.0 \pm 3.4$ & $6.3 \pm 4.1$ & $4.3 \pm 3.1$ & $0.001^{*}$ \\
\hline Number of patients & 10 & 10 & 10 & \\
\hline Predialysis HGF (pg/ml) & $150.5(44.2-681)$ & $93(42-231.8)$ & $156.7(88-201)$ & 0.2 \\
\hline Number of patients & 6 & 6 & 7 & \\
\hline
\end{tabular}

Data are expressed as mean SD or IQR (25-75).

$\mathrm{BHD}=$ bicarbonate hemodialysis; $\mathrm{HDF}=$ online hemodiafiltration; $\mathrm{C}-\mathrm{RP}$ = C-reactive protein; $\mathrm{BUN}=$ blood urea nitrogen; spKT/V = single-pool KT/V; HGF $=$ hepatocyte growth factor.

${ }^{*} \mathrm{HDF}$ versus BHD.

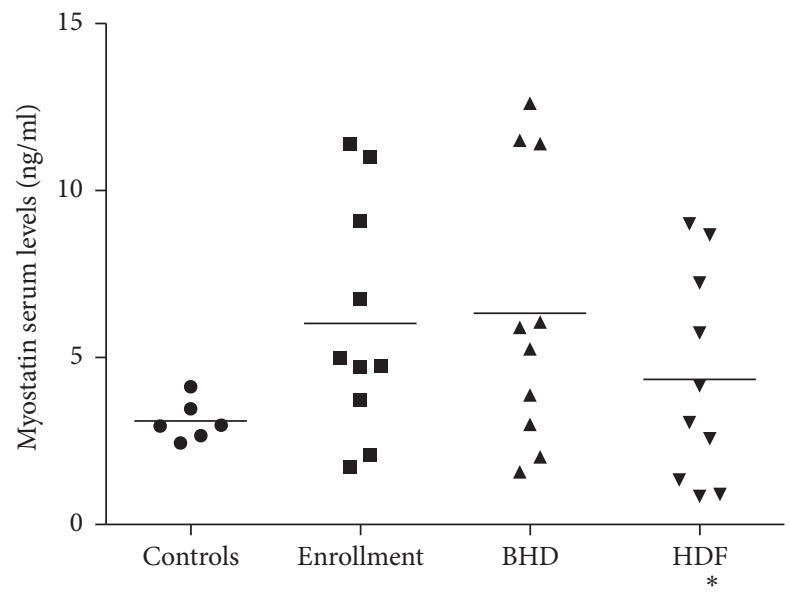

FIGURE 1: Effect of different dialysis modalities on myostatin serum levels. $\mathrm{BHD}=$ bicarbonate hemodialysis; $\mathrm{HDF}=$ online hemodiafiltration. At the enrollment all the patients were on $\mathrm{BDH}$ for at least 6 months. ${ }^{*} p<0.05$ versus enrollment and BHD.

88-201) $\mathrm{pg} / \mathrm{ml}, p=0.2]$, whereas serum myostatin levels significantly decreased [BHD $6.3 \pm 4.1$ versus HDF $4.3 \pm$ $3.1 \mathrm{ng} / \mathrm{ml}, p=0.001$ (Figure 1)]. The differences in myostatin levels from BHD to HDF were $-1.4 \pm 1.7 \mathrm{ng} / \mathrm{ml}$ for group 1 and $-1.1 \pm 2.3 \mathrm{ng} / \mathrm{ml}$ for group $2, p=0.9$.

Therefore, starting with BHD or HDF did not seem to have a significant impact on HD modality-induced changes in myostatin levels.

\section{Discussion}

With this study we have demonstrated that in regular HD patients the use of different HD modalities could have different effects on myokine balance. In particular online HDF, a convective dialytic technique associated with better depuration capacity and a higher rate of cytokine removal, significantly reduced myostatin levels, without affecting HGF [12].

This is an important consideration since myostatin and HGF seem to exert opposite effects on the modulation of muscle cell metabolism. In fact, myostatin, where serum levels are elevated in CKD and in cachectic states, may act as a negative regulator of muscle growth and differentiation $[13,14]$, whereas HGF exerts proliferative and differentiative stimuli on muscle cells. So, it is possible that these molecules play a role in the pathogenesis of muscle wasting in HD, also by a feedback mechanism. However, our findings highlighted that only in patients undergoing online HDF myostatin and HGF resulted in being inversely correlated. Similarly, we found that only during HDF myostatin and HGF revealed a relationship with nutritional parameters. In particular, myostatin, as expected by its role as negative metabolic regulator, was inversely related to transferrin, a parameter strongly associated with a good nutritional status [15]. On the opposite, HGF was directly related to BMI, which, in turn, seems to be protective in HD patients [16].

Therefore, it seems that high-volume online HDF treatment could be able to restore a more "physiological" condition in which myostatin and HGF are counterregulated and correlated with nutritional parameters.

This preliminary finding, which surely needs further confirmations, together with the evidence that BHD and HDF exert different effects on myostatin and HGF levels, might have a great clinical relevance. Indeed, in the past years research activity has been addressed to the development of new dialysis modalities, mainly convective techniques, which could improve clearance of uremic toxins, also providing higher clearances of middle molecules, such as inflammatory cytokines [17].

In this view, high-volume online HDF, using high biocompatible membranes and ultrapure dialysate, has been 
found to be more efficient than standard BHD, resulting in being also associated with a lower mortality rate [18-20]. Our data provide new and intriguing evidence on the potential advantages of convective strategies, showing that the use of HDF, through the modulation of myokine levels, might also promote a state of better muscle trophism, as previously only hypothesized [21]. This is particularly relevant considering that, in spite of several efforts to understand the underlying mechanisms of these high-risk conditions, currently there are no established strategies to prevent and manage malnutrition and wasting syndrome in HD.

On the other hand, there is a growing interest in the study of myokines, in particular myostatin, as therapeutic targets for the treatment of skeletal muscle wasting/atrophy under diverse clinical settings, including denervation, AIDS, cancer, diabetes, and chronic heart failure [22]. Indeed, several experimental studies demonstrated that pharmacological inhibition of myostatin by genetic interference or specifically designed antibody was associated with increased muscle mass and improved metabolic profile [23, 24], suggesting that myokine modulation might realistically represent a promising approach to prevent and treat muscle wasting.

We are aware that our data present some weaknesses. In fact, although the crossover design of our study was intended to reduce interindividual heterogeneity, since each patient served as his own control, the small number of patients enrolled constitutes a limitation of this study.

Moreover, we did not perform a structured assessment of the nutritional status of our patients, also considering that the treatment periods were probably too short to appreciate any significant clinical change in muscle mass or strength.

\section{Conclusions}

Malnutrition and muscle wasting syndrome remain unsolved problems in HD patients, strictly related to the elevated morbidity and mortality distinctive of this patient population.

Therefore, the understanding of underlying mechanisms could provide new therapeutic targets and strategies to face these conditions. With the preliminary findings of our study we offer a new point of view on this issue, showing that the use of different HD modalities, in particular convective-based techniques, can influence myokine profile.

In light of this evidence, we hypothesize that HDmediated myokine modulation might potentially represent a novel approach to the prevention and treatment of HDrelated muscle wasting syndrome. Prospective studies with a larger number of patients are needed to confirm these data and evaluate their clinical impact.

\section{Conflicts of Interest}

The authors have no conflicts of interest to declare.

\section{Acknowledgments}

The authors thank the Scientific Direction of the Fondazione IRCCS Policlinico San Matteo of Pavia for the financial (Progetto di Ricerca Corrente) and scientific support.

\section{References}

[1] A. Rashid Qureshi, A. Alvestrand, J. C. Divino-Filho et al., "Inflammation, malnutrition, and cardiac disease as predictors of mortality in hemodialysis patients," Journal of the American Society of Nephrology, vol. 13, no. 1, pp. S28-S36, 2002.

[2] X. H. Wang and W. E. Mitch, "Mechanisms of muscle wasting in chronic kidney disease," Nature Reviews Nephrology, vol. 10, no. 9, pp. 504-516, 2014.

[3] S.-J. Lee, "Regulation of muscle mass by myostatin," Annual Review of Cell and Developmental Biology, vol. 20, pp. 61-86, 2004.

[4] J. Jespersen, M. Kjaer, and P. Schjerling, "The possible role of myostatin in skeletal muscle atrophy and cachexia," Scandinavian Journal of Medicine and Science in Sports, vol. 16, no. 2, pp. 74-82, 2006.

[5] N. F. Gonzalez-Cadavid, W. E. Taylor, K. Yarasheski et al., "Organization of the human myostatin gene and expression in healthy men and HIV-infected men with muscle wasting," Proceedings of the National Academy of Sciences of the United States of America, vol. 95, no. 25, pp. 14938-14943, 1998.

[6] K. A. Reardon, J. Davis, and R. M. I. Kapsa, "Myostatin, insulinlike growth factor-1, and leukemia inhibitory factor mRNAs are upregulated in chronic human disuse muscle atrophy," Muscle and Nerve, vol. 24, no. 7, pp. 893-899, 2001.

[7] F. Galimi, M. F. Brizzi, and P. M. Comoglio, "The hepatocyte growth factor and its receptor," Stem Cells, vol. 11, supplement 2, pp. 22-30, 1993.

[8] S. Hayashi, H. Aso, K. Watanabe et al., "Sequence of IGF-I, IGF-II, and HGF expression in regenerating skeletal muscle," Histochemistry and Cell Biology, vol. 122, no. 5, pp. 427-434, 2004.

[9] L. Zhang, J. Pan, Y. Dong et al., "Stat3 activation links a C/EBP $\delta$ to myostatin pathway to stimulate loss of muscle mass," Cell Metabolism, vol. 18, no. 3, pp. 368-379, 2013.

[10] D. S. Han, Y. M. Chen, S. Y. Lin et al., "Serum myostatin levels and grip strength in normal subjects and patients on maintenance haemodialysis," Clinical Endocrinology, vol. 75, no. 6, pp. 857-863, 2011.

[11] C. Libetta, P. Esposito, C. Martinelli et al., "Hepatocyte Growth Factor (HGF) and hemodialysis: physiopathology and clinical implications," Clinical and Experimental Nephrology, vol. 20, no. 3, pp. 371-378, 2016.

[12] G. Thomas and B. L. Jaber, "Convective therapies for removal of middle molecular weight uremic toxins in end-stage renal disease: a review of the evidence," Seminars in Dialysis, vol. 22, no. 6, pp. 610-614, 2009.

[13] S. Yano, A. Nagai, M. Isomura et al., "Relationship between blood myostatin levels and kidney function:shimane CoHRE study," PLoS ONE, vol. 10, no. 10, Article ID e0141035, 2015.

[14] H. Q. Han, X. Zhou, W. E. Mitch, and A. L. Goldberg, "Myostatin/activin pathway antagonism: molecular basis and therapeutic potential," International Journal of Biochemistry and Cell Biology, vol. 45, no. 10, pp. 2333-2347, 2013.

[15] K. Kalantar-Zadeh, M. Kleiner, E. Dunne et al., "Total iron-binding capacity-estimated transferrin correlates with the nutritional subjective global assessment in hemodialysis patients," American Journal of Kidney Diseases, vol. 31, no. 2, pp. 263-272, 1998.

[16] R. Pellicano, B. J. Strauss, K. R. Polkinghorne, and P. G. Kerr, "Longitudinal body composition changes due to dialysis," 
Clinical Journal of the American Society of Nephrology, vol. 6, no. 7, pp. 1668-1675, 2011.

[17] N. Meert, S. Eloot, M.-A. Waterloos et al., "Effective removal of protein-bound uraemic solutes by different convective strategies: a prospective trial," Nephrology Dialysis Transplantation, vol. 24, no. 2, pp. 562-570, 2009.

[18] P. Bolasco, P. Alteiri, S. Andrulli et al., "Convection versus diffusion in dialysis: an Italian prospective multicentre study," Nephrology Dialysis Transplantation, vol. 18, supplement 7, pp. vii50-vii54, 2003.

[19] A. Davenport, S. A. E. Peters, M. L. Bots et al., "Higher convection volume exchange with online hemodiafiltration is associated with survival advantage for dialysis patients: the effect of adjustment for body size," Kidney International, vol. 89, no. 1, pp. 193-199, 2016.

[20] G. Jean, J.-M. Hurot, P. Deleaval, B. Mayor, and C. Lorriaux, "Online-haemodiafiltration vs. conventional haemodialysis: a cross-over study," BMC Nephrology, vol. 16, article 70, 2015.

[21] C. Basile, "The effect of convection on the nutritional status of haemodialysis patients," Nephrology Dialysis Transplantation, vol. 18, supplement 7, pp. vii46-vii49, 2003.

[22] V. Dutt, S. Gupta, R. Dabur, E. Injeti, and A. Mittal, "Skeletal muscle atrophy: potential therapeutic agents and their mechanisms of action," Pharmacological Research, vol. 99, pp. 86-100, 2015.

[23] S. Cohen, J. A. Nathan, and A. L. Goldberg, "Muscle wasting in disease: molecular mechanisms and promising therapies," Nature Reviews Drug Discovery, vol. 14, no. 1, pp. 58-74, 2014.

[24] J.-P. G. Camporez, M. C. Petersen, A. Abudukadier et al., "Antimyostatin antibody increases muscle mass and strength and improves insulin sensitivity in old mice," Proceedings of the National Academy of Sciences of the United States of America, vol. 113, no. 8, pp. 2212-2217, 2016. 


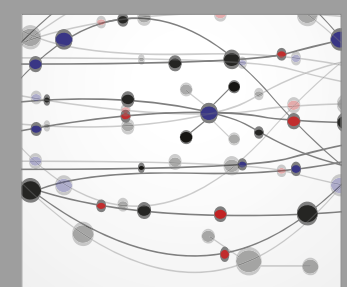

The Scientific World Journal
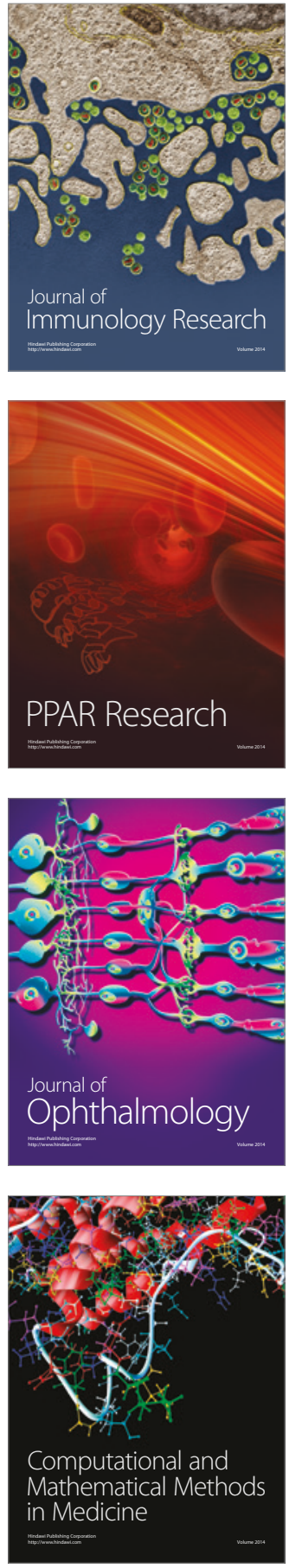

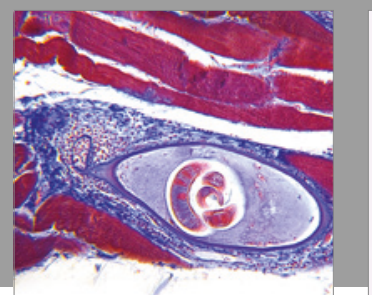

Gastroenterology Research and Practice
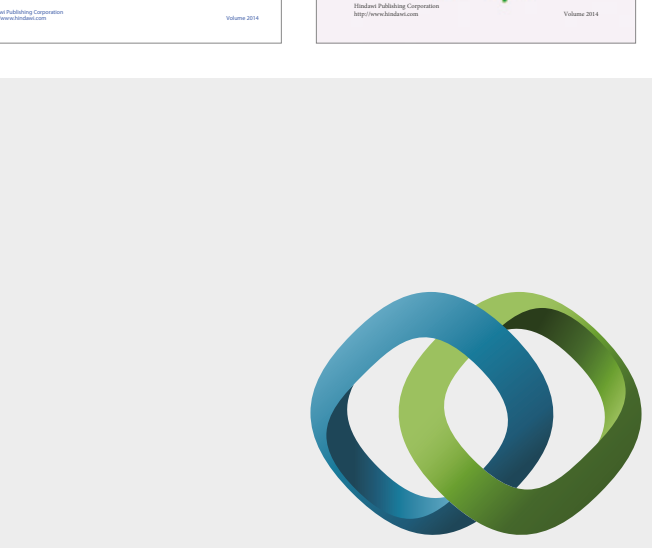

\section{Hindawi}

Submit your manuscripts at

https://www.hindawi.com
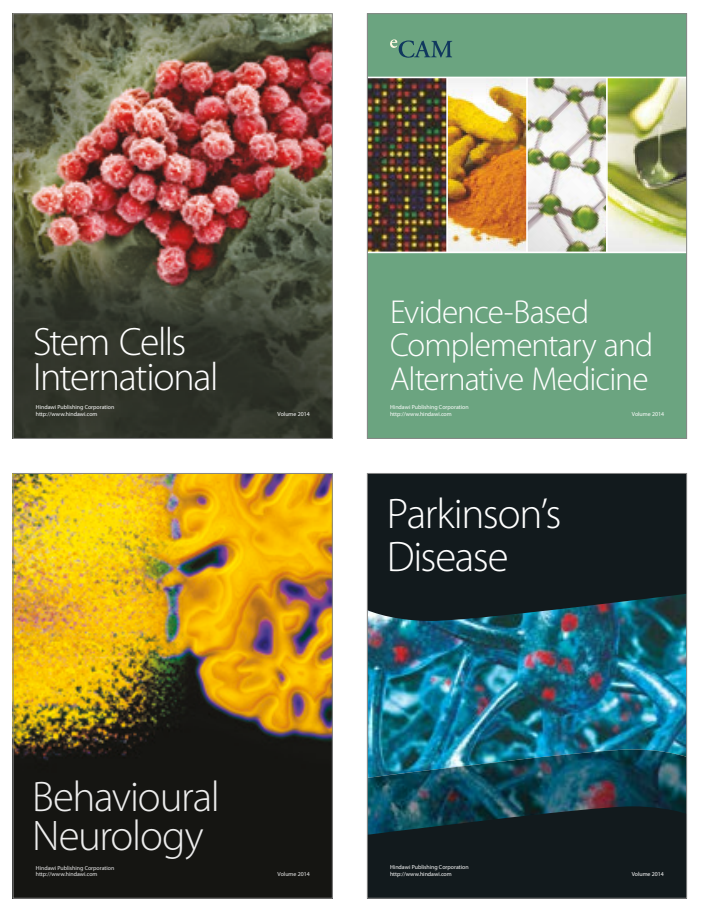
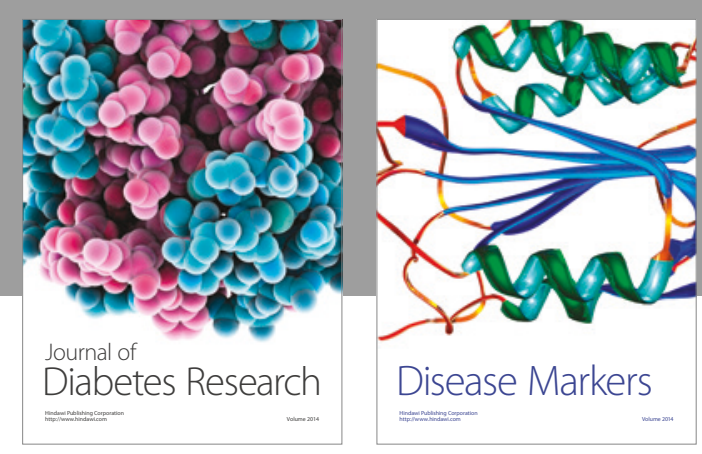

Disease Markers
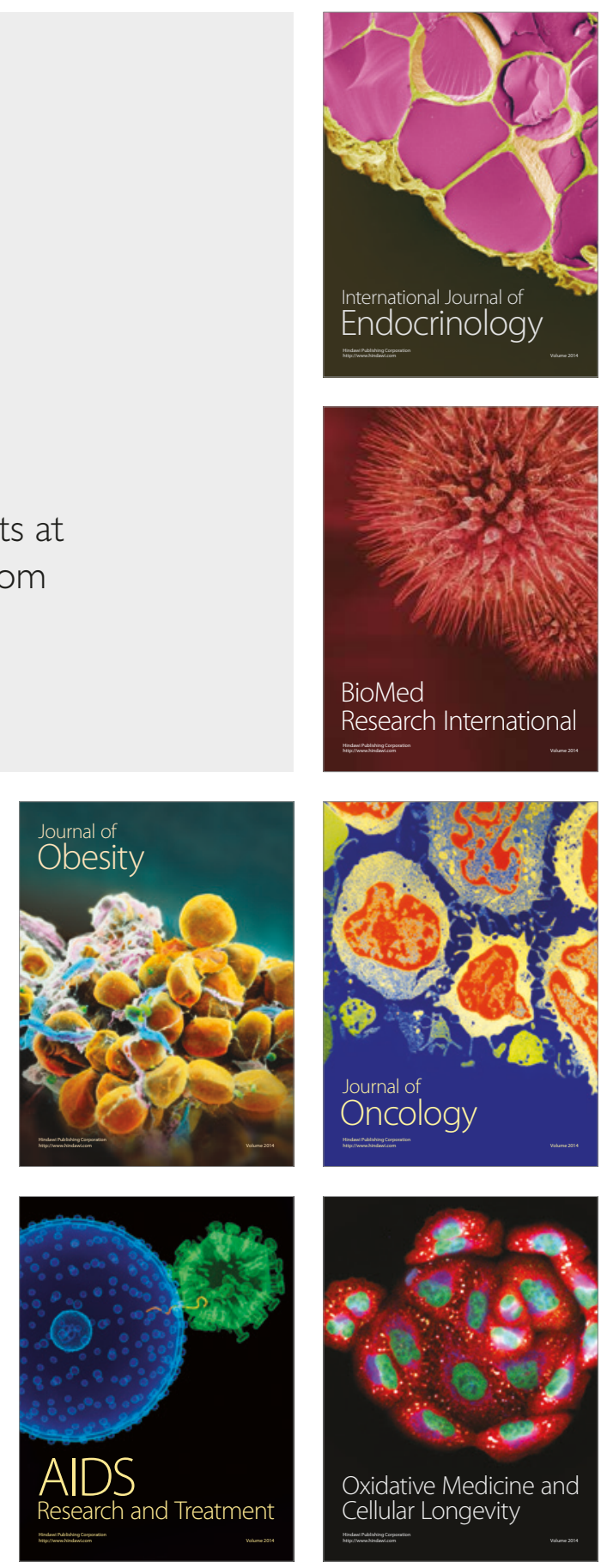